\title{
XMM-Newton view of galaxy pairs: activation of quiescent black holes?`
}

\author{
E. Jiménez-Bailón ${ }^{1}$, N. Loiseau ${ }^{2}$, M. Guainazzi ${ }^{2}$, G. Matt ${ }^{1}$, D. Rosa-González ${ }^{3}$, E. Piconcelli ${ }^{4}$, and M. Santos-Lleó ${ }^{2}$ \\ 1 Dipartimento di Fisica di l'Universita Roma 3, via della Vasca Navale 84, 00146 Roma, Italy \\ e-mail: ejimenez@fis.uniroma3.it \\ 2 XMM-Newton Science Operations Center, ESAC, ESA, Apartado 50727, 28080 Madrid, Spain \\ 3 Instituto Nacional de Astrofísica Optica y Electrónica, Luis Enrique Erro No. 1. Tonantzintla, Puebla, C.P. 72840, México \\ 4 Osservatorio Astronomico di Roma (INAF), via Frascati 33, 00040 Monteporzio Catone, Italy
}

Received 17 November 2006 / Accepted 23 March 2007

\section{ABSTRACT}

\begin{abstract}
We report on XMM-Newton observations of three nearby galaxy pairs, AM0707-273, AM1211-465, and AM2040-674. All six galaxies were previously classified as HII galaxies based on optical and IR spectroscopic analysis. All galaxies were detected with XMM-Newton and each member was isolated and analyzed independently. The X-ray spectra reveal strong evidence of AGN activity in the NE member of AM1211-465 pair. We measured a luminosity of $1.94_{-0.15}^{+0.11} \times 10^{42} \mathrm{erg} / \mathrm{s}$ in the $2-10 \mathrm{keV}$ band and the presence of a neutral FeK $\alpha$ line with a confidence level of $98.8 \%$. The high $n_{\mathrm{H}}$ value, $2.2 \pm 0.2 \times 10^{22} \mathrm{~cm}^{-2}$, would explain the misclassification of the source. Marginal evidence of AGN nature was found in the X-ray spectra of AM1211-465SW and AM0707-273E. The X-ray emission of the three remaining galaxies can be explained by starburst activity.
\end{abstract}

Key words. galaxies: active - galaxies: nuclei - galaxies: general - X-rays: galaxies - X-rays: general

\section{Introduction}

Based on optical surveys, only about $0.1 \%$ of all quasars observed have one, or more, nearby quasar companion at the same redshift (e.g., Kochanek 1995; Hewett et al. 1998). However, there is a growing number of identifications of true pairs of quasars or as excellent candidates (see Hennawi et al. 2006 on SDSS results). Although highly redshifted pairs tend to be interpreted as the result of gravitational lensing by dense objects in the line of sight, many of them cannot be explained in this way, and even the spectral features that were supposed to indicate that they were one and the same object have been interpreted as a common feature of all quasars (Mortlock et al. 1999). For these cases the most reasonable interpretation is that their activity is the result of their interaction. Even though the interaction of two large galaxies is not always strong enough to create new supermassive black holes in the cores, it is plausible that the interaction can refuel the quiescent black holes hosted by the majority of known galaxies.

Assuming that most of the galaxies host a supermassive black hole (Kormendy et al. 1997), the key question in our understanding of AGN formation and evolution would be the mechanisms that trigger this nuclear activity. Galaxies interactions are thought to be effective in driving the gas from the outer parts of the galaxy into the circumnuclear region through loss of the angular momentum induced by tidal forces (Jogee et al. 2005). The inflow of gas could be intercepted by the starburst activity in the circumnuclear region or could continue falling into the nucleus feeding the back hole (Shlosman et al. 2005). However, no direct

^ Based on observations obtained with XMM-Newton, an ESA science mission with instruments and contributions directly funded by ESA Member States and NASA. causal links between the degree of the interaction and the AGN activity have been found up to now.

Recent X-ray detections show that many interacting galaxies classified as non-AGN are in fact active (e.g. Maiolino et al. 2003). These findings prove that optical spectroscopy is sometimes inefficient in revealing the presence of an AGN. The X-ray study of a small sample of binary quasars has revealed that all of them suffer some degree of absorption and at least half of them resulted to be Compton-thick (Komossa et al. 2003; Ballo et al. 2004; Guainazzi et al. 2005). These findings show that a significant number of AGN pairs might be missed by optical surveys due to heavy obscuration affecting one (or both) of the members.

In this paper we have studied the X-ray emission of three pairs of nearby interacting galaxies observed with XMM-Newton: AM0707-273, AM1211-465, and AM2040-674. In the following section we summarize the data analysis of the observations. All the studied galaxies can be classified as having some kind of activity in the central regions. In Sect. 3, we discuss the origin of this nuclear emission as either starburst or AGN). The main conclusions of this work are presented in Sect. 4.

\section{Observations and data reduction of the XMM-Newton data}

The three pairs of galaxies (AM0707-273, AM1211-465, and AM2040-674) were selected from the sample of Sekiguchi \& Wolstencroft (1992) of interacting doubles of comparably sized galaxies, i.e. with relative sizes of members to be within a factor of two. The galaxies also present a disturbed appearance in optical and/or IR images proving the interaction. The pairs present enough projected separation distance to be spatially resolved by XMM-Newton. All six galaxies in the sample are 


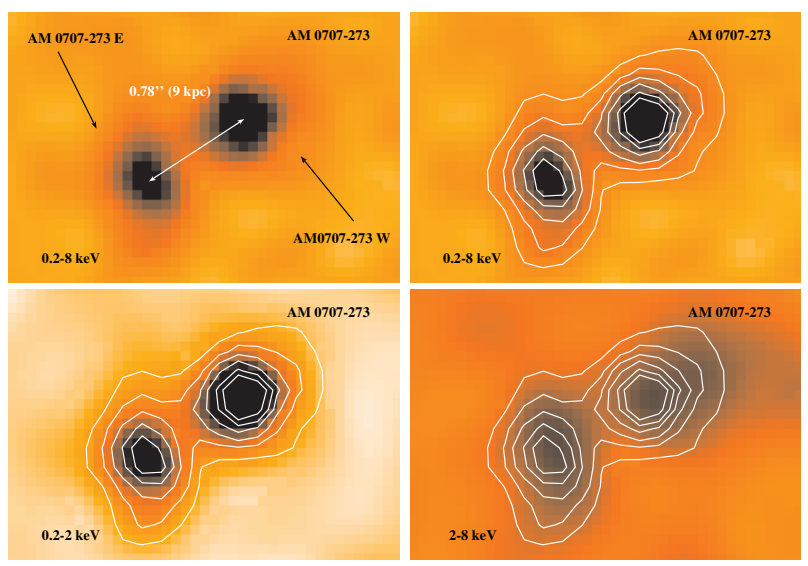

Fig. 1. Smoothed pn images of AM 0707-273 on a logarithmic scale from 0 to 150 counts. From top to bottom and left to right: a) location and separation distance of the sources superimposed on the smoothed $0.2-8 \mathrm{keV}$ image. b) $0.2-8 \mathrm{keV}$ surface brightness contours superimposed on the smoothed $0.2-2 \mathrm{keV}$ image. Contour levels are at 0.03 , $0.2,1,7,45$, and 285 counts. c) $0.2-2 \mathrm{keV}$ surface brightness contours superimposed on the smoothed $2-8 \mathrm{keV}$ image. d) $2-8 \mathrm{keV}$ surface brightness contours superimposed on the smoothed $0.2-8 \mathrm{keV}$ image.

classified as HII galaxies based on optical and IR data (Sekiguchi \& Wolstencroft 1992).

The details of the XMM-Newton (Jansen et al. 2001) observations of the pairs of galaxies are summarized in Table 1 . The raw data were processed using the standard Science Analysis Systems, SAS, v.7.0.0. (Gabriel et al. 2004). The most updated calibration files available in July 2006 were used for the reduction of the data. We removed time intervals corresponding to high background using the method described in Piconcelli et al. (2004). According to the epaplot $S A S$ task, no significant pile up was detected in none of the studied sources.

\subsection{Imaging analysis}

Smoothed images of the galaxy pairs were generated using the asmooth SAS task applied to the $p n$ images. We applied the adaptive convolution technique, designed for Poissonian images, with $S / N=10$. Figures $1 \mathrm{a}, 3 \mathrm{a}$, and 5a show the $p n$ $0.2-8 \mathrm{keV}$ smoothed images of each of the targets. All six galaxies were clearly detected in the whole energy band and the $X M M-N e w t o n$ spatial resolution allowed us to disentangle the two members in all pairs. In the figures, we have named each pair member and the separation distances are also indicated. In Table 2, we collate the locations, the separation distance, both in arcsec and in kpc for all pairs, the redshift, and the Galactic equivalent hydrogen column obtained from Dickney \& Lockman (1990). We also produced images in the soft $(0.2-2 \mathrm{keV})$ and in the hard (2-8 keV) bands.

Radial profiles were produced in the $0.2-8 \mathrm{keV}$ band for both AM0707-273 members. Despite of their proximity, which prevents us from reaching firm conclusions, both sources seem to be extended when compared with the point-like profile of MCG-6-30-15 (see Fig. 2). In fact, the AM0707-273E component presents a more extended profile than its companion. This extended nature of the emission gives hints to the merging process. As seen in Fig. 1, both galaxies show intense nuclear emission in the hard band.

In the case of AM1211-465, the radial profile indicates that both sources are point-like in the broad $0.2-8 \mathrm{keV}$ energy band (see Fig. 4). Both galaxies are detected in the hard energy band.
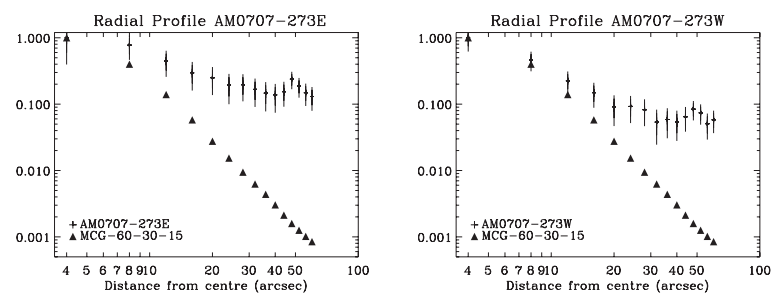

Fig. 2. Radial profile of AM 0707-273E (left panel), i.e. integrated net counts normalized versus distance from the center of the galaxy, and AM 0707-273W (right panel) in the $0.2-8 \mathrm{keV}$ energy band. Radial profiles of the point-like source MCG-60-30-15 in the same energy bands are also plotted for comparison. In both profiles, a local maximum of the normalized net counts due to presence of the companion is observed around $50^{\prime \prime}$.

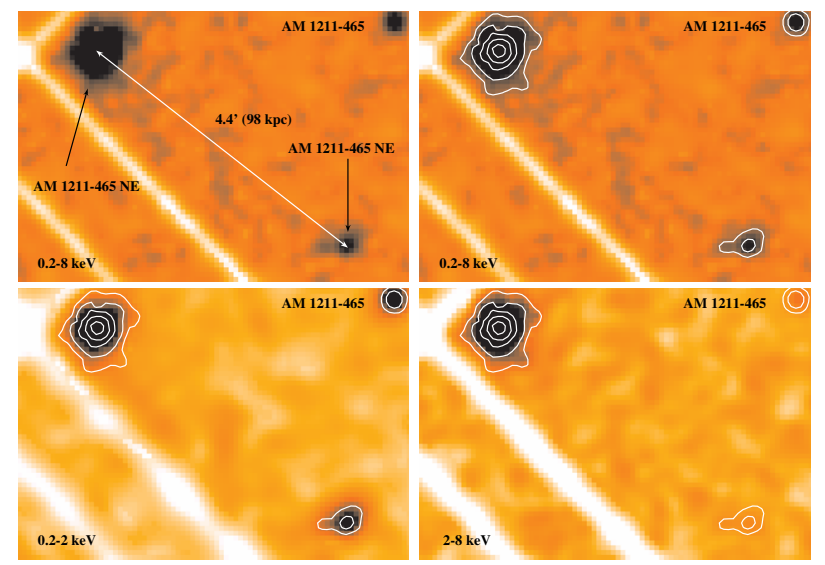

Fig. 3. Smoothed $p n$ images of AM 1211-465 on a logarithmic scale from 0 to 350 counts. From top to bottom and left to right: a) location and separation distance of the sources superimposed on the smoothed $0.2-8 \mathrm{keV}$ image. b) $0.2-8 \mathrm{keV}$ surface brightness contours superimposed on the smoothed $0.2-2 \mathrm{keV}$ image. Contour levels are at 7,10 , $1,20,50$, and 150 counts. c) $0.2-8 \mathrm{keV}$ surface brightness contours superimposed on the smoothed $2-8 \mathrm{keV}$ image. d) $0.2-8 \mathrm{keV}$ surface brightness contours superimposed on the smoothed $0.2-8 \mathrm{keV}$ image.

The AM1211-465NE hard-band image reveals the presence of a very intense X-ray source. The images show a region of diffuse emission between the sources $10 \sigma$ above the background, strengthening the possibility of an effective encounter between the galaxies, even though its apparent separation is high, $98 \mathrm{kpc}$. Unfortunately, its weakness and the proximity of the edge of the CCD to the sources do not allow more accurate analysis of the shape of this diffuse emission.

Finally, the two galaxies of AM2040-674 are visible in the soft energy band but AM2040-674N was undetectable in the hard energy band. The radial profile (Fig. 6) is not conclusive, due to the proximity of the sources $\left(\sim 50^{\prime \prime}\right)$ and the low number of counts detected for the northern member. However, marginal hints of extended emission are shown in the AM2040- 674-S radial profile.

\subsection{Spectral analysis}

We extracted the EPIC spectra for the sources with enough counts. The MOS 1 and MOS 2 spectra were combined in order to increase the signal-to-noise, and the resulting spectra were analyzed together with the $p n$ one. All $p n$ and $M O S 1 / 2$ spectra were grouped such that each bin contains at least 20 counts in order to apply the modified $\chi^{2}$ minimization technique 
Table 1. General properties and details of the observations of the galaxy pairs.

\begin{tabular}{|c|c|c|c|c|c|c|}
\hline Target & Obs. ID & Obs. date & $\begin{array}{l}\text { Obs. Mode } \\
\text { (1) }\end{array}$ & $\begin{array}{l}\text { Filter } \\
\text { (2) }\end{array}$ & $\begin{array}{l}p n \text { Exp. } \\
(3) \\
\text { ks }\end{array}$ & $\begin{array}{l}\text { Net pn CR } \\
(4) \\
\text { count/s }\end{array}$ \\
\hline $\begin{array}{l}\text { AM 0707-273 } \\
A M 0707-273 E \\
A M 0707-273 W\end{array}$ & 0300930201 & $2005-09-27$ & $\overline{F F}$ & $\bar{M}$ & $\begin{array}{l}11.33 \\
11.33\end{array}$ & $\begin{array}{l}(2.26 \pm 0.16) \times 10^{-2} \\
(2.99 \pm 0.18) \times 10^{-2}\end{array}$ \\
\hline $\begin{array}{l}\text { AM 1211-465 } \\
\text { AM 1211-465NE } \\
A M 1211-465 S W\end{array}$ & 0300930101 & $2005-09-28$ & FF & $\bar{T}$ & $\begin{array}{l}12.88 \\
5.48\end{array}$ & $\begin{array}{l}(2.55 \pm 0.05) \times 10^{-2} \\
(2.8 \pm 0.3) \times 10^{-2}\end{array}$ \\
\hline $\begin{array}{l}\text { AM 2040-674 } \\
A M 2040-674 N \\
A M 2040-674 S\end{array}$ & 0300930301 & $2005-09-24$ & FF & $\mathrm{T}$ & $\begin{array}{l}9.83 \\
12.22\end{array}$ & $\begin{array}{l}(7.1 \pm 1.0) \times 10^{-3} \\
(1.17 \pm 0.11) \times 10^{-2}\end{array}$ \\
\hline
\end{tabular}

Notes: (1) Observations Modes: FF: Full Frame Mode; (2) Observations Filters: T: Thin, M: Medium; (3) Useful pn exposure; (4) Net pn count rates in the $0.2-10 \mathrm{keV}$ band.

Table 2. Locations and separation distances for each of the galaxy pairs.

\begin{tabular}{|c|c|c|c|c|c|c|}
\hline \multirow[t]{2}{*}{ Target } & \multicolumn{2}{|c|}{ Coordinates J2000 } & \multicolumn{2}{|c|}{ Separation } & \multirow{2}{*}{$\begin{array}{l}\text { Redshift } \\
\text { (1) }\end{array}$} & \multirow{2}{*}{$\begin{array}{l}n_{\mathrm{H}}^{\text {Gal }} \\
10^{20} \mathrm{~cm}^{-2}\end{array}$} \\
\hline & RA & Dec & arcmin & $\mathrm{kpc}$ & & \\
\hline $\begin{array}{l}\text { AM 0707-273E } \\
\text { AM 0707-273W }\end{array}$ & $\begin{array}{l}070949 . ' 6 \\
070946 . ' 6 \\
\end{array}$ & $\begin{array}{l}-273435 \\
-273409 \\
\end{array}$ & $0: 78$ & 9 & $\begin{array}{l}{ }^{a} 0.00989 \pm\left(2 \times 10^{-5}\right) \\
{ }^{b} 0.00989 \pm\left(2 \times 10^{-5}\right)\end{array}$ & 1.7 \\
\hline $\begin{array}{l}\text { AM 1211-465NE } \\
\text { AM 1211-465SW }\end{array}$ & $\begin{array}{lll}12 & 14 & 13^{\prime \prime} 0 \\
12 & 14 & 52^{\prime \prime} 4\end{array}$ & $\begin{array}{l}-471345 \\
-471629\end{array}$ & 4.4 & 98 & $\begin{array}{ll}{ }^{c} & 0.01849 \pm\left(1.2 \times 10^{-4}\right) \\
{ }^{d} & 0.01848 \pm\left(1.7 \times 10^{-4}\right)\end{array}$ & 8.4 \\
\hline $\begin{array}{l}\text { AM 2040-674N } \\
\text { AM 2040-674S }\end{array}$ & $\begin{array}{l}204519 \text { I. }^{\prime} 8 \\
204521^{\prime \prime} 0\end{array}$ & $\begin{array}{l}-673216 \\
-673304\end{array}$ & 0.82 & 34 & $\begin{array}{l}{ }^{e} 0.03249 \pm\left(9 \times 10^{-5}\right) \\
e^{e} 0.03263 \pm\left(9 \times 10^{-5}\right)\end{array}$ & 4.3 \\
\hline
\end{tabular}

Notes: (1) Redshift values obtained from $\left({ }^{a}\right)$ Theureau at al. (2005); $\left({ }^{b}\right)$ Wong (2006); $\left(^{c}\right)$ Strauss et al. (1992); $\left({ }^{d}\right)$ Fouque et al. (1992); $\left({ }^{e}\right)$ Sekiguchi \& Wolstencroft (1992).
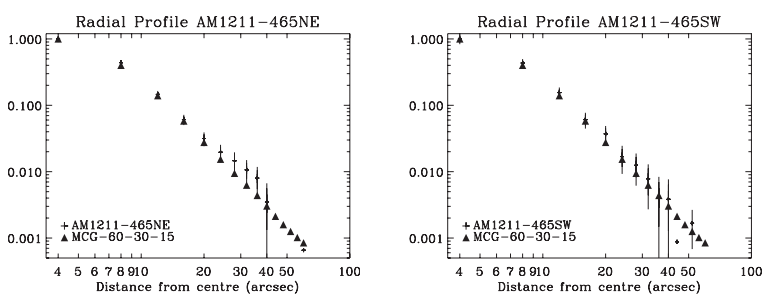

Fig. 4. Radial profile of AM 1211-465NE (left panel) and AM 1211$465 \mathrm{SW}$ (right panel) in the $0.2-8 \mathrm{keV}$ energy band. Radial profiles of the point-like source MCG-60-30-15 in the same energy bands are also plotted for comparison.

(Kendall et al. 1973) in the spectral analysis. For all cases, the source extraction region was circular with a radius chosen to maximize the signal-to-noise ratio and to keep it from overlapping the extraction region of the companion source. The background extraction regions were also circular, placed in the same CCD of the targets and in a field free from other contamination sources. Two of the pairs, AM 0707-273 and AM 2040674 , show separation between galaxies on the order of $50^{\prime \prime}$. For these cases, the extraction radii are $20^{\prime \prime}$. The fraction of encircled energy for the $p n$ detector within $30^{\prime \prime}$ is measured to be higher than $80 \%$ (XMM-Newton User's Handbook V2.4); therefore, we did not expect a significant contamination from the companion source in the spectrum. Only for one of the galaxies, AM 2040-674N, we did not detect enough counts to perform a spectral analysis. The spectral analysis was performed using XSPECv.12.2.0 (Arnaud 1996). The quoted errors for the fit parameters referred to the $90 \%$ confidence level (i.e. $\Delta \chi^{2}=2.71$, Avni 1976). We assumed a Hubble constant of $70 \mathrm{~km} \mathrm{~s}^{-1}$ and a flat cosmology with $\Omega_{M}, \Omega_{\Delta}=(0.3,0.7)$ (Bennett et al. 2003).

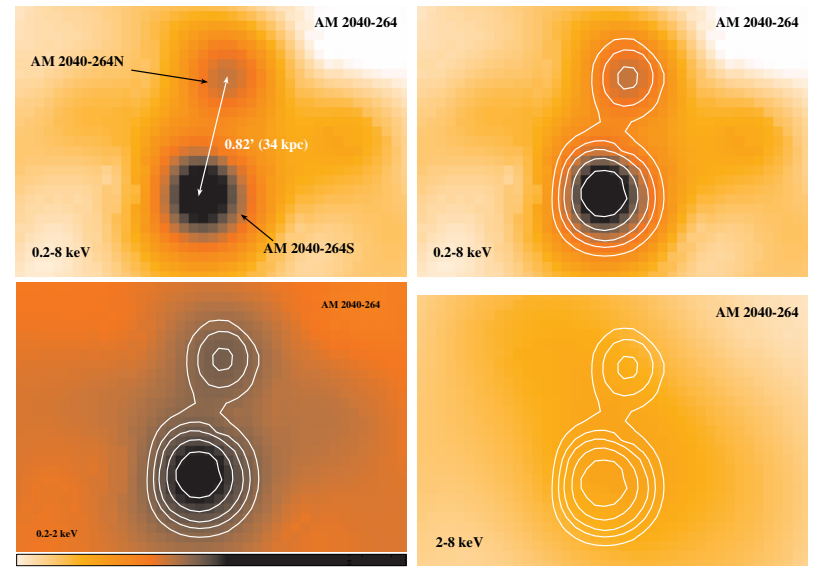

Fig. 5. Smoothed $p n$ images of AM 2040-674 on a logarithmic scale from 0 to 150 counts. From top to bottom and left to right: a) location and separation distance of the sources superimposed on the smoothed $0.2-8 \mathrm{keV}$ image. b) $0.2-8 \mathrm{keV}$ surface brightness contours superimposed on the smoothed $0.2-2 \mathrm{keV}$ image. Contour levels are at 0.0003 , $0.003,0.03,0.3$, and 3 counts. c) $0.2-8 \mathrm{keV}$ surface brightness contours superimposed on the smoothed $2-8 \mathrm{keV}$ image. d) $0.2-8 \mathrm{keV}$ surface brightness contours superimposed on the smoothed $0.2-8 \mathrm{keV}$ image.

To elucidate the nature of the sources, several spectral models have been tested for each source spectrum. Table 3 summarizes all the tested models, the values of their parameters, and the goodness of the fits for each source. Single-component models (power law or thermal mekal emission) did not provide a satisfactory fit for any of the spectra analyzed, excluding the AM1211-465SW spectrum, satisfactorily fitted with a single power law (see below). We also applied two-component models 
Table 3. Results of the spectral analysis of the five galaxies.

\begin{tabular}{|c|c|c|c|c|c|c|c|c|}
\hline \multirow[t]{2}{*}{ Target } & \multirow{2}{*}{$\begin{array}{l}\text { Model } \\
\text { (1) }\end{array}$} & $\overline{n_{\mathrm{H}}}$ & $\bar{\Gamma} \Gamma / k T$ & $\overline{n_{\mathrm{H}}}$ & $\overline{k k T}$ & $E W(\mathrm{Fe}$ line $)$ & Significance & Goodness \\
\hline & & \multicolumn{2}{|l|}{$10^{21} \mathrm{~cm}^{-2}$} & $10^{21} \mathrm{~cm}^{-2}$ & $\mathrm{keV}$ & \multicolumn{3}{|l|}{$\mathrm{eV}$} \\
\hline \multirow[t]{5}{*}{ AM 0707-273-E } & A & $1.1 \pm 0.6$ & $1.9_{-0.2}^{+0.3}$ & - & - & - & - & 33 for 22 d.o.f. \\
\hline & $\mathrm{B}$ & $\leq 0.3$ & $6_{-2}^{+4}$ & - & - & - & - & 35 for 22 d.o.f. \\
\hline & $\mathbf{C}$ & $\sim 0$ & $1.2 \pm 0.2$ & $10.4_{-1.9}^{+1.1}$ & $0.143_{-0.002}^{+0.07}$ & $<2400$ & $89 \%$ & 19 for 20 d.o.f. \\
\hline & $\mathrm{D}$ & $\sim 0$ & $1.2_{-0.7}^{+0.2}$ & $9_{-8}^{+200^{-1.9}}$ & $0.16_{-0.05}^{+0.5002}$ & - & - & 21 for 20 d.o.f. \\
\hline & $\mathrm{E}$ & $<1.2$ & $\geq 7^{-0.1}$ & $\sim 0$ & $0.85_{-0.16}^{+0.25}$ & - & - & 20 for 20 d.o.f. \\
\hline \multirow[t]{5}{*}{ AM 0707-273-W } & A & $2.8_{-0.8}^{+0.7}$ & $3.1_{-0.3}^{+0.4}$ & - & - & - & - & 56 for 30 \\
\hline & $\mathrm{B}$ & 0.15 & 3 & - & - & - & - & 87 for 30 d.o.f. \\
\hline & $\mathbf{C}$ & $1.3_{-1.1}^{+1.9}$ & $2.2_{-0.5}^{+0.8}$ & & $0.43_{-0.2}^{+0.15}$ & $<9000$ & $80 \%$ & 40 for 27 d.o.f. \\
\hline & $\mathrm{D}$ & $2.6_{-1.5}^{+4.1}$ & $2.7_{-0.3}^{-0.6}$ & $3.0 \pm 0.9$ & $0.45_{-0.10}^{+0.17}$ & - & - & 46 for 27 d.o.f. \\
\hline & $\mathrm{E}$ & $0.7_{-0.4}^{+1.5}$ & $3.6_{-1.0}^{+1.2}$ & $\sim 0$ & $0.59_{-0.12}^{+0.08}$ & - & - & 49 for 27 d.o.f. \\
\hline \multirow[t]{5}{*}{ AM 1211-465-NE } & $\mathrm{A}$ & 12 & 1.3 & - & - & - & - & 531 for 214 d.o.f. \\
\hline & $\mathrm{B}$ & 11 & $>80$ & - & - & - & - & 547 for 214 d.o.f. \\
\hline & $\mathbf{C}$ & $22 \pm 2$ & $1.63 \pm 0.10$ & $1.2_{-0.4}^{+1.3}$ & $0.52_{-0.19}^{+0.11}$ & $110 \pm 70$ & $98.8 \%$ & 228 for 209 d.o.f. \\
\hline & $\mathrm{D}$ & $22_{-3}^{+5}$ & $1.64_{-0.13}^{+0.12}$ & $4_{-2}^{+3}$ & $0.25_{-0.14}^{+0.29}$ & - & - & 241 for 211 d.o.f. \\
\hline & $\mathrm{E}$ & $13.1_{-0.5}^{+1.4}$ & $\geq 65$ & $\sim 0$ & $0.40_{-0.06}^{+0.10}$ & - & - & 294 for 212 d.o.f. \\
\hline \multirow[t]{5}{*}{ AM 1211-465-SW } & A & $\sim 0$ & $1.7 \pm 0.2$ & - & - & - & - & 22 for 21 d.o.f. \\
\hline & B & $\sim 0$ & $8_{-3}^{+16}$ & - & - & - & - & 26 for 21 d.o.f. \\
\hline & $\mathbf{C}$ & $\sim 0$ & $1.0 \pm 0.5$ & $8.0_{-1.4}^{+7}$ & $<0.2$ & $<3000$ & $72 \%$ & 14 for 18 d.o.f. \\
\hline & $\mathrm{D}$ & $\sim 0$ & $0.3_{-0.3}^{+0.5}$ & $<7^{-1.4}$ & $0.6_{-0.4}^{+1.6}$ & - & - & 14 for 18 d.o.f. \\
\hline & $\mathrm{E}$ & 8 & $>12$ & $7.9_{-1.3}^{+1.6}$ & $<0.3$ & - & - & 14 for 18 d.o.f. \\
\hline \multirow[t]{5}{*}{ AM 2040-674-S } & A & $1.1_{-0.6}^{+0.8}$ & $3.2_{-0.4}^{+0.6}$ & - & - & - & - & 22 for 12 d.o.f. \\
\hline & B & $\sim 0^{-0.6}$ & $3^{-0.4}$ & - & - & - & - & 64 for 13 d.o.f. \\
\hline & $\mathbf{C}$ & $\sim 0$ & $1.9_{-0.2}^{+0.4}$ & $\sim 0$ & $0.33_{-0.06}^{+0.18}$ & $<4 \times 10^{6}$ & $50 \%$ & 9 for 11 d.o.f. \\
\hline & $\mathrm{D}$ & $\sim 0$ & $1.4^{-0.2}$ & $\sim 0$ & $0.5^{-0.00}$ & - & - & 22 for 11 d.o.f. \\
\hline & $\mathrm{E}$ & $\sim 0$ & $7_{-4}^{+40}$ & $\sim 0$ & $0.32_{-0.05}^{+6}$ & - & - & 10 for 11 d.o.f. \\
\hline
\end{tabular}

Notes: (1) Model A: wabs*pwlw; Model B: wabs*mekal; Model C: wabs*pwlw+wabs*mekal; Model D: wabs*pwlw+wabs*bremsstrahlung; Model E: wabs*(mekal+mekal). wabs represents photoelectric absorption (Morrison \& McCammon 1983) and $p w l w$ a power law emission model. The best fit model is marked in boldface.

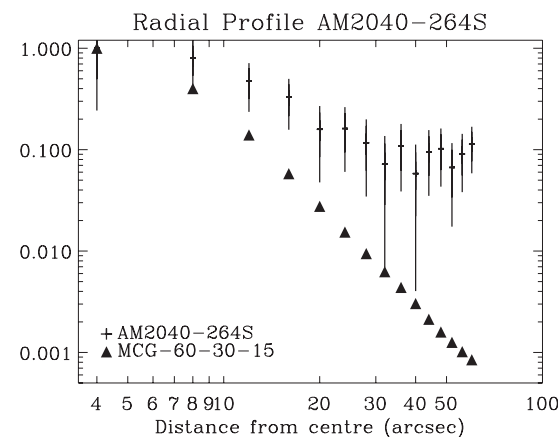

Fig. 6. Radial profile of AM 2040-674S in the $0.2-8 \mathrm{keV}$ energy band. Radial profile of the point-like source MCG-60-30-15 in the same energy bands are also plotted for comparison.

to all spectra: a power law and two different thermal models (mekal and bremsstrahlung). The power-law component accounts for either a possible AGN and, in the case of starburst origin of the emission, for the joint contribution of the emission from X-ray binaries and a less significant contribution of Compton radiation that originated in the interaction between FIR photons with high-energy cosmic rays from SN ejecta. The thermal model accounts for the joint contribution of the compact stellar-like X-ray sources and the warm and ionized gas in superwinds. The values of the best-fit parameters span wide ranges. In particular, the power-law index varies from object to object in the 1.0 to 2.2 range, when only the best-fit model is taken into account. The flatter power laws are found for both galaxies in AM1211-465 and for AM0707-273E. In the two remaining cases, i.e. AM0707-273W and AM2040-674N, the index of the power law is compatible with values $\$ 2$.

The excess observed in the soft energy band can be satisfactorily fitted with thermal emission models. Similar $\chi^{2}$ values are obtained for the two tested models, mekal and bremsstrahlung for both members of AM1211-465 and AM0707-273E. The $\chi^{2}$ values for the mekal and bremsstrahlung models differ slightly for AM0707-273W and AM2040-674S. Although the temperatures of the thermal components that largely vary from object to object, the values are compatible with the ones found in nearby interacting systems, which are well-fitted with temperatures ranging from $\sim 0.15$ to $\sim 0.9 \mathrm{keV}$ (Jenkins et al. 2004, and references therein). The soft X-ray emission of merging systems is usually well-fitted with multi-temperature thermal components (Jenkins et al. 2004, and references therein). Nonetheless, the addition of a second thermal component to account for the soft emission did not significantly improve any of the spectral fits.

The abundances were initially fixed to the solar value. In general, when they were left free to vary, no bounded values were found. Only in the case of AM1211-465NE, does a different metallicity from the solar one significantly improve the fit, $Z=0.030_{-0.018}^{+0.03}$, which makes this model physically indistinguishable from the bremsstrahlung model. The fitting analysis 
Table 4. Absorbed fluxes and unabsorbed luminosities for the six galaxies.

\begin{tabular}{lllll}
\hline \hline Target & $\begin{array}{l}\text { Flux }(0.5-2 \mathrm{keV}) \\
10^{-14} \mathrm{erg} \mathrm{cm}^{-2} \mathrm{~s}^{-1}\end{array}$ & $\begin{array}{l}\text { Flux (2-10 keV) } \\
10^{-14} \mathrm{erg} \mathrm{cm}^{-2} \mathrm{~s}^{-1}\end{array}$ & $\begin{array}{l}\text { Luminosity }(0.5-2 \mathrm{keV}) \\
10^{41} \mathrm{erg} \mathrm{s}^{-1}\end{array}$ & $\begin{array}{l}\text { Luminosity (2-10 keV) } \\
10^{41} \mathrm{erg} \mathrm{s}^{-1}\end{array}$ \\
\hline AM 0707-273-E & $2.89_{-1.6}^{+0.11}$ & $6.9_{-2}^{+1.0}$ & $33.6_{-20}^{+1.3}$ & $0.15_{-0.04}^{+0.02}$ \\
pwlw & $1.93(70 \%)$ & $6.9(\sim 100 \%)$ & $0.05(-)$ & $0.15(\sim 100 \%)$ \\
mekal & $0.96(30 \%)$ & $8 \times 10^{-4}(-)$ & $33.6(\sim 100 \%)$ & $3 \times 10^{-4}(-)$ \\
AM 0707-273-W & $4.3_{-2}^{+0.2}$ & $3.7_{-1.5}^{+0.6}$ & $0.375_{-0.17}^{+0.017}$ & $0.082_{-0.03}^{+0.013}$ \\
pwlw & $2.9(70 \%)$ & $3.6(99 \%)$ & $0.17(45 \%)$ & $0.08(98 \%)$ \\
mekal & $1.4(30 \%)$ & $0.05(1 \%)$ & $0.205(55 \%)$ & $1.3 \times 10^{-3}(2 \%)$ \\
\hline AM 1211-465-NE & $15.6_{-3}^{+0.7}$ & $214_{-17}^{+13}$ & $10.6_{-2}^{+0.5}$ & $19.4_{-1.5}^{+1.1}$ \\
pwlw & $11(70 \%)$ & $212(\sim 100 \%)$ & $9.8(90 \%)$ & $19.4(\sim 100 \%)$ \\
Raymond-Smith & $4.6(30 \%)$ & $3 \times 10^{-3}(-)$ & $0.8(\sim 10 \%)$ & $0.02(-)$ \\
AM 1211-465-SW & $3.4_{-2}^{+0.5}$ & $12_{-4}^{+2}$ & $65_{-40}^{+10}$ & $0.90_{-0.3}^{+0.15}$ \\
pwlw & $2.0(60 \%)$ & $12(\sim 100 \%)$ & $0.2(-)$ & $0.90(\sim 100 \%)$ \\
mekal & $1.4(40 \%)$ & $5 \times 10^{-4}(-)$ & $65(\sim 100 \%)$ & $7 \times 10^{-5}(-)$ \\
\hline AM 2040-674-N ${ }^{\dagger}$ & 0.9 & 1.8 & 0.28 & 0.43 \\
AM 2040-674-S & $1.9_{-0.2}^{+0.3}$ & $1.7_{-0.7}^{+0.3}$ & $0.83_{-0.09}^{+0.13}$ & $0.41_{-0.17}^{+0.07}$ \\
pwlw & $1.2(60 \%)$ & $1.7(\sim 100 \%)$ & $0.51(60 \%)$ & $0.41(\sim 100 \%)$ \\
Raymond-Smith & $0.7(40 \%)$ & $3 \times 10^{-3}(-)$ & $0.32(40 \%)$ & $9 \times 10^{-4}(-)$ \\
\hline
\end{tabular}

Notes: $\dagger$ Upper limits fluxes and luminosities calculated using the net $p n$ count rate measured, i.e. $7.1 \times 10^{-3} \mathrm{c} / \mathrm{s}$, and assuming an absorbed $n_{\mathrm{H}}=$ $1 \times 10^{21} \mathrm{~cm}^{-2} \Gamma=1.8$ power law emission model.

reveals that similar values of the $\chi_{v}^{2}$ are found for the two different types of thermal models applied.

A neutral absorbing gas-material component was tested to separately affect each of the spectral components (power law and thermal emission). In some cases, we found values of the $N_{\mathrm{H}}$ close to zero, so we removed the absorbing component from the testing model. Only in the case of AM1211-465NE, was a large equivalent hydrogen column, $n_{\mathrm{H}}=2.2 \pm 0.2 \times 10^{22} \mathrm{~cm}^{-2}$, measured. A large absorption, $n_{\mathrm{H}}=1.04_{-0.19}^{+0.11} \times 10^{22} \mathrm{~cm}^{-2}$, affecting the thermal component was measured in AM0707-273E. For the rest of the objects, in the cases in which an absorbing component is required, the values found are on the order of $10^{21} \mathrm{~cm}^{-2}$. The absence of absorbing material for AM2040-674S could indicate that the merging process is not effectively obscuring the systems.

We also systematically tested the presence of neutral $\mathrm{K} \alpha$ iron line. This feature is expected to be present in highlyabsorbed AGN, and therefore its occurrence would provide evidence of the presence of Seyfert nucleus. A narrow Gaussian line fixed to $6.4 \mathrm{keV}$ significantly improves the $\chi^{2}$ only in the AM1211-465 NE spectrum with a confidence level of $\gtrsim 98.8 \%$, according to the F-test. A lower confidence level value, but still relatively high $(\sim 90 \%)$, was found for the galaxy AM0707-273E. The equivalent width $(E W)$ measured was $110 \pm 70 \mathrm{eV}$, fully in agreement with the mean $E W$ of a sample of quasars observed with XMM-Newton (Jiménez-Bailón et al. 2005). Upper limits to the $E W$ are calculated in the rest of the objects.

Finally, we tested a double thermal mekal emission model to explain the whole energy spectrum. The one with the lower temperature associated with the heated gas related to the superwinds and the higher temperature one associated with the collisionally excited gas created by stellar winds and $\mathrm{SNe}$.

The double thermal model is not the best model in any of the cases; however, the results are still statically acceptable. Bounded measurements of the temperature can be derived for AM0707-273W and AM2040-674S, $\left(3.6_{-1.0}^{+1.2} \mathrm{keV}\right.$ and $7_{-4}^{+40} \mathrm{keV}$, respectively). These values are consistent with those found for starburst galaxies (Persic \& Rephaeli 2002; Cappi et al. 1999). For the rest of the objects, i.e. AM0707-273E, AM1211-465NE and AM1211-465SW, only lower limits for the temperature were derived.

Fluxes and luminosities in soft and hard energy bands derived from the best-fit models are given in Table 4 . The luminosities of the galaxies in the hard band $(2-10 \mathrm{keV})$ range from $\sim 8 \times$ $10^{39}$ to $\sim 2 \times 10^{42} \mathrm{ergs} / \mathrm{s}$. In comparison with several samples of LINER and HII galaxies, these luminosities are in general above the mean values. Ho et al. (2001) calculated using Chandra a mean $L_{2-10 \mathrm{keV}}=2 \times 10^{38} \mathrm{erg} / \mathrm{s}$ for a sample of LINER and composite LINER/HII galaxies. Recently González-Martín et al. (2006) calculated a mean $L_{2-10 \mathrm{keV}}$ of $3.8 \times 10^{39} \mathrm{erg} / \mathrm{s}$ for a sample of 51 optically selected LINER, covering the range between $1.2 \times 10^{38}-1.5 \times 10^{42} \mathrm{erg} / \mathrm{s}$.

The X-ray light curves in different energy bands, i.e. $0.3-10 \mathrm{keV}, 0.5-2 \mathrm{keV}$, and $2-10 \mathrm{keV}$, were produced for the five objects with a sufficient number of detected counts. No signs of variability within the observation were observed for any of the objects.

\section{Discussion}

It is accepted that merging activity induces starforming episodes. However, we still do not fully understand the connection between the origin of the energy budget and the properties of the involved galaxies, such as the pair separation, the shapes of the galaxies, or a possible presence of an AGN. In this section, we present the X-ray properties of the three interacting systems and discuss the nature of their emission. We also consider the results in the framework of the scenario of black hole activation in merging systems (Mortlock et al. 1999). Finally, we compare our results with the very few other cases where nearby binary AGN were detected. 

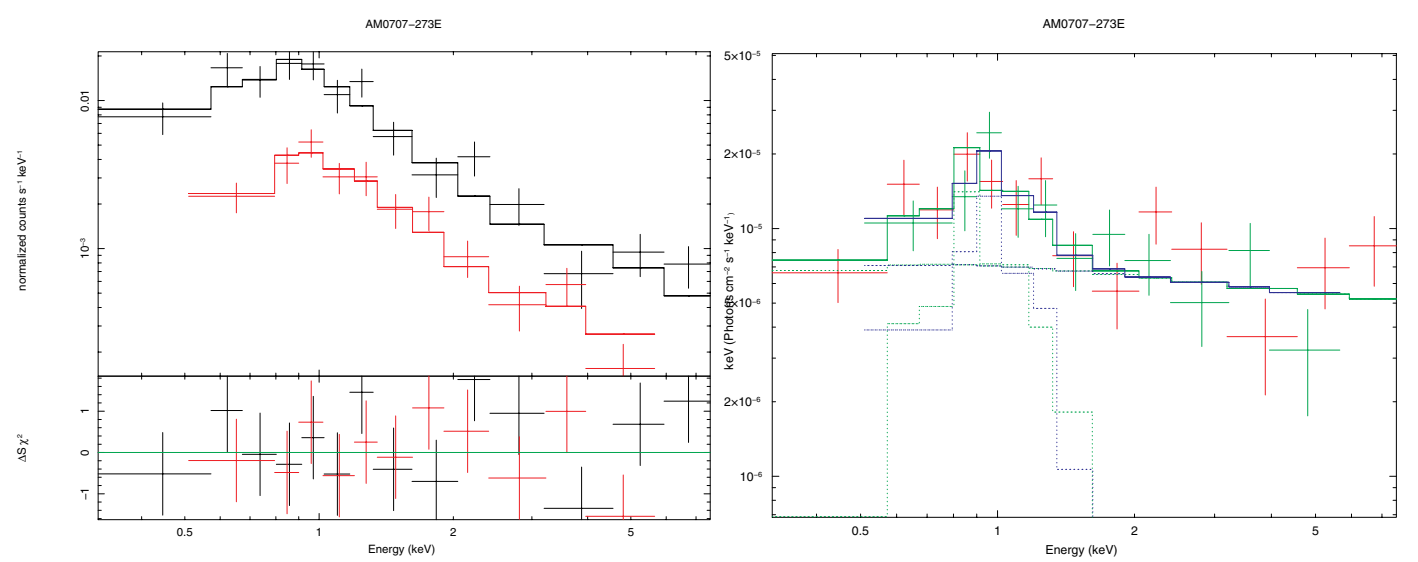

Fig. 7. Observed spectrum, best fit-model, and residuals of $A M 0707-273-E$ on the left and the unfolded spectrum and the model in Ef(E) on the right.
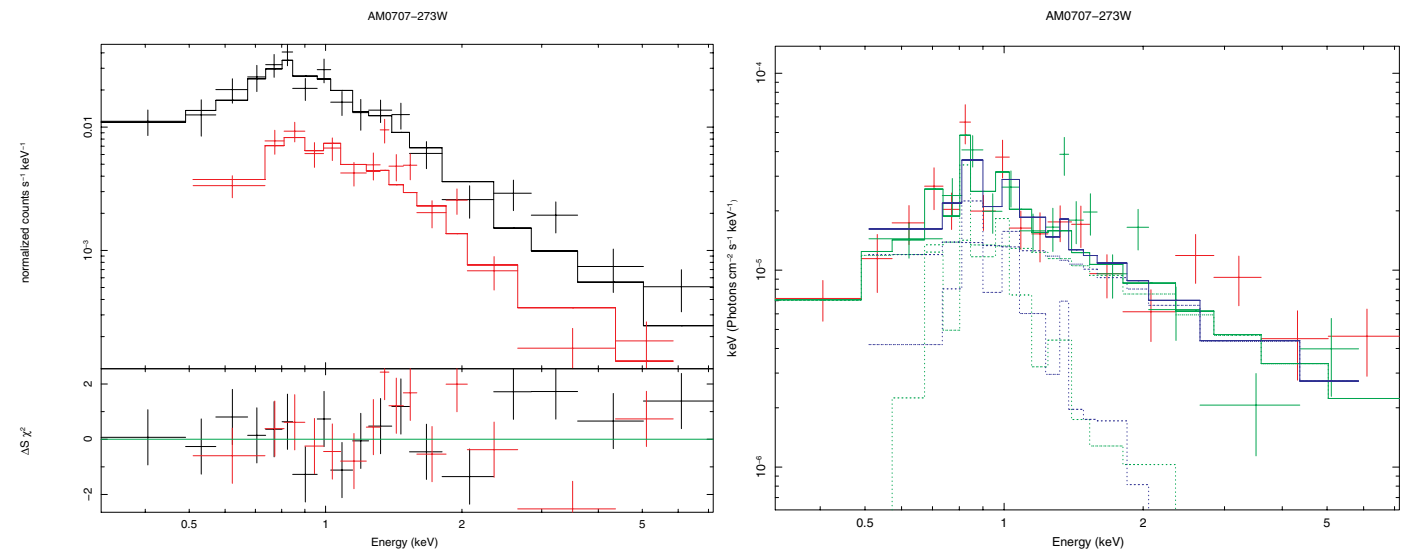

Fig. 8. Observed spectrum, best-fit model, and residuals of $A M$ 0707-273-W on the left and the unfolded spectrum and the model in Ef(E) on the right.
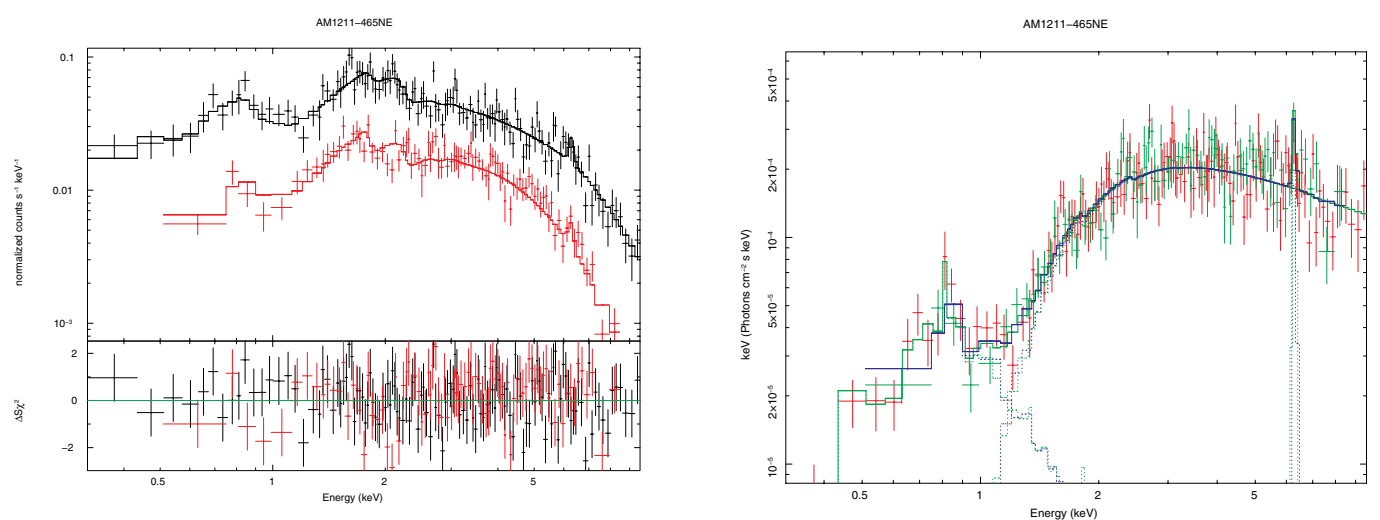

Fig. 9. Observed spectrum, best-fit model, and residuals of $A M$ 1211-465-NE on the left, and the unfolded spectrum and the model in Ef(E) on the right.

\subsection{Starburst versus AGN as the origin of the nuclear X-ray emission}

Among the six studied sources, only AM1211-465NE can be unambiguously classified as an AGN. The imaging analysis supports a point-like nature. The X-ray luminosity in the hard band, $1.94_{-0.15}^{+0.11} \times 10^{42} \mathrm{ergs} / \mathrm{s}$, is difficult to explain with only starformation activity. The value found for the power law index, $1.63 \pm 0.10$, also agrees well with the mean values observed for AGN. Interestingly, the soft excess is preferably fitted with a mekal model, but with a very low metallicity, $0.030_{-0.018}^{+0.03}$, or a bremsstrahlung component. In general, starforming regions tend to be fitted with higher metallicities (e.g. Strickland et al. 2004), due to the enrichment of the environment by type-II SN, (e.g. Arnett 1995). However, low metallicity values have been measured (Strickland \& Stevens 2000) in starforming regions. This low value is probably due to an artifact of fitting the multiphase plasma emission with single-temperature models. The measurement of the equivalent hydrogen column of the neutral absorbing material, $2.2 \pm 0.2 \times 10^{22} \mathrm{~cm}^{-2}$, is compatible with typical values found for Seyfert 2 galaxies. If this neutral material is located in the AGN itself, its origin could be related to the 

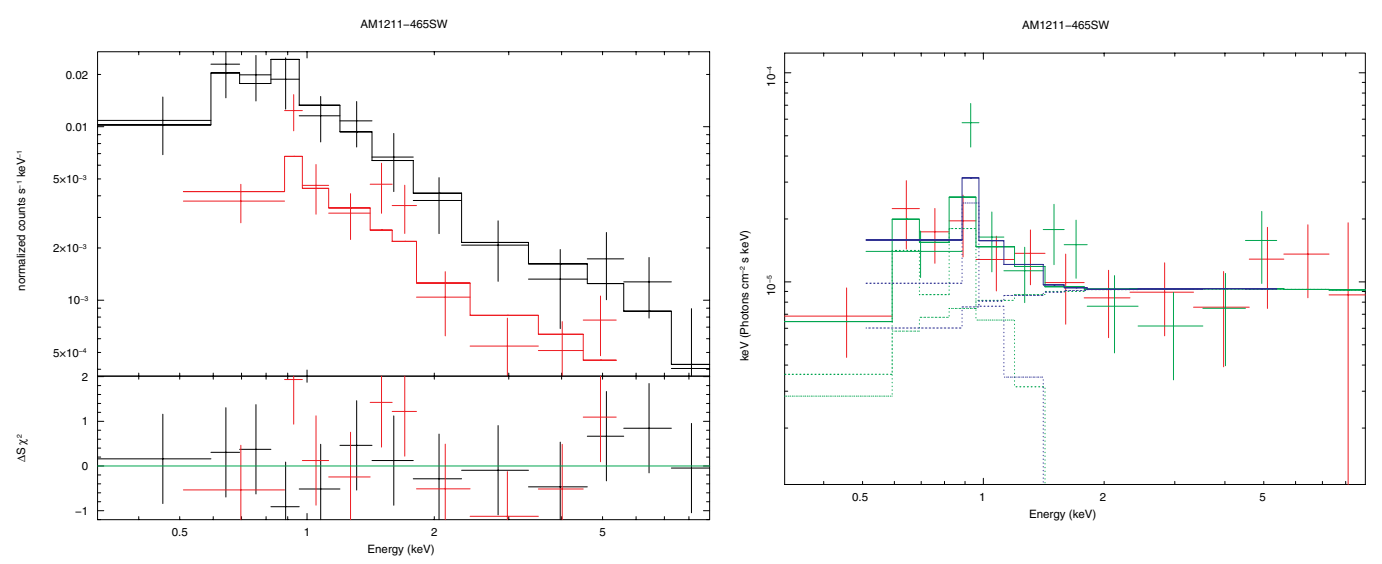

Fig. 10. Observed spectrum, best-fit model, and residuals of $A M 1211-465-S W$ on the left and the unfolded spectrum and the model in Ef(E) on the right.
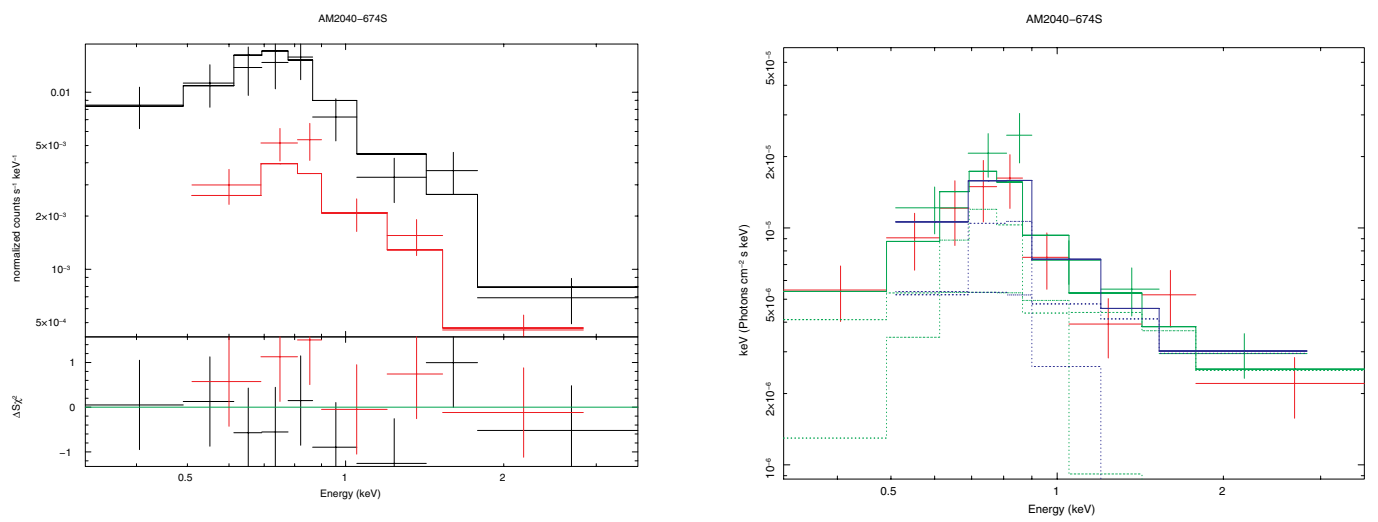

Fig. 11. Observed spectrum, best-fit model, and residuals of $A M$ 2040-674-S on the left and the unfolded spectrum and the model in Ef(E) on the right.

shielding torus. Another possibility is that the merging process is causing gas inflows that are effectively obscuring the nuclear emission. This highly obscuring material could be the reason for the classification of AM1211-465NE as a HII galaxy (Corbett et al. 2003; Sekiguchi \& Wolstencroft 1992) using optical and IR data. Finally, the detection ( $98.8 \%$ according to the F-test) of a neutral $\mathrm{Fe} \mathrm{K} \alpha$ emission line reinforces the AGN origin of the nuclear emission of AM1211-465NE. Allowing the energy of line to vary did not improve the goodness of the fit. However we measured a value of $6.34_{-0.2}^{+0.14} \mathrm{keV}$, incompatible with the ionized iron emission line typically at $6.7 \mathrm{keV}$. In fact, if the energy of the line is fixed to this value, the addition of this component does not improve the fit.

On the other hand, the spatial and spectral results on the both components of the AM2040-674 pair and AM0707-273W indicates that their X-ray emission can be easily explained with star-formation processes. The image of AM2040- 674N shows a diffuse emission visible only in the soft band. Too few counts are detected for a spectral analysis to be possible, but upper limits on the soft and hard X-ray fluxes were derived by assuming an absorbed $\left(n_{\mathrm{H}}=1 \times 10^{21} \mathrm{~cm}^{-2}\right)$ power-law $(\Gamma=1.8)$ emission model (see Table 4). For the other two objects, AM0707273W and AM2040-67S, the hard and soft X-ray luminosities are lower than $10^{40} \mathrm{erg} / \mathrm{s}$, in the range of HII and normal galaxies. Moreover, both X-ray spectra can be fitted with a double thermal component with reasonable values for the temperatures, although these fits are significantly worse than power law plus thermal emission models. We derived the star-formation rate
(SFR) of these two galaxies using the empirical relationship with the $2-10 \mathrm{keV}$ luminosity of Grimm et al. (2003): $L_{2-10 \mathrm{keV}}=$ $6.7 \times 10^{39} \mathrm{SFR}$. The values obtained are $1.2 \mathrm{M}_{\odot} / \mathrm{yr}$ for AM0707273W and 6.0 $M_{\odot} / \mathrm{yr}$ for AM2040-674S. An upper limit, $S F R<$ $6.4 M_{\odot} / y r$, was calculated for AM2040-674N. According to Kennicutt (1998), the FIR luminosities derived from these values of the $\operatorname{SFR}\left(\operatorname{SFR}\left(>0.1 M_{\odot}\right)=\frac{L_{\mathrm{FIR}}}{5.8 \times 10^{9} L_{\odot}} M_{\odot} \mathrm{yr}^{-1}\right)$ are within the same order of magnitude or even lower than the observed with IRAS. In particular, the $L_{\mathrm{FIR}}$ measured for the AM 2040-674 pair is $2.6 \times 10^{-10} \mathrm{erg} \mathrm{cm}^{-1} \mathrm{~s}^{-1}$ and the $L_{\mathrm{FIR}}$ derived from the SFR of AM2040-674S is $0.6 \times 10^{-10} \mathrm{erg} \mathrm{cm}^{-1} \mathrm{~s}^{-1}$. For the AM0707273 , the observed $L_{\mathrm{FIR}}$ is $8 \times 10^{-10} \mathrm{erg} \mathrm{cm}^{-1} \mathrm{~s}^{-1}$, while the $L_{\mathrm{FIR}}$ derived from the calculated $S F R$ is $3.5 \times 10^{-10} \mathrm{erg} \mathrm{cm}^{-1} \mathrm{~s}^{-1}$ (see below for the contribution of AM0707-273E).

The spectral properties of the E member of AM0707-273 are compatible with both an AGN and powerful starburst origin. The imaging analysis favors the second possibility because the radial profile suggests an extended emission. The temperature of the mekal plasma, $k T=0.143_{-0.002}^{+0.07} \mathrm{keV}$, is compatible with the typical values obtained for AGN. On the other hand, the index of the power law, $1.2 \pm 0.2$ is lower than the typical values of AGN. Likewise, although the luminosity measured for the galaxy, $L_{0.3-10 \mathrm{keV}} \sim 2 \times 10^{42} \mathrm{erg} / \mathrm{s}$ is high compared with the X-ray luminosities of normal or HII galaxies, it is in the soft band where its contribution is higher. This high luminosity could be an artifact of the high absorbing column associated to the thermal component, $n_{\mathrm{H}}=1 \times 10^{22} \mathrm{~cm}^{-2}$. In fact, we calculated a lower absorption, $n_{\mathrm{H}} \sim 2 \times 10^{21} \mathrm{~cm}^{-2}$, using the $\mathrm{H} \alpha / \mathrm{H} \beta$ ratio 
obtained from Sekiguchi \& Wolstencroft (1992). We therefore fixed the index of the power law to 1.8 (compatible with mean values of $\Gamma$ found in both AGN and starforming galaxy spectra), leaving the rest of the parameters free. Although the value of $n_{\mathrm{H}}$ associated to the thermal component decreased, the soft band luminosity, $L_{0.5-2 \mathrm{keV}} \sim 7 \times 10^{41} \mathrm{erg} / \mathrm{s}$, is still high. However, in the hard band, the measured luminosity is only on the order of $10^{40} \mathrm{erg} / \mathrm{s}$. Although it is a luminous object in the X-ray band, its nature could also be compatible with intense starburst activity. If this is the case, the SFR derived from the $2-10 \mathrm{keV}$ luminosity of the source (Grimm et al. 2003) would be $2.2 M_{\odot} / \mathrm{yr}$.

Finally, the results of the analysis of AM1211-465SW are more ambiguous. The imaging analysis indicates a point-like emission. The power law is very flat, $\Gamma=1.0 \pm 0.5$, and the total luminosity is $L_{0.3-10 \mathrm{keV}} \sim 4 \times 10^{42} \mathrm{erg} / \mathrm{s}$, even higher than the value measured for AM1211-465NE. We also tried to fix the index of the power law to 1.8. The value of $n_{\mathrm{H}}$ associated to the thermal component decreased slightly, but the luminosity in the $0.3-10 \mathrm{keV}$ band remains unvaried. In the hard band, the measured luminosity is also high, $\sim 1 \times 10^{41} \mathrm{erg} / \mathrm{s}$, higher than the luminosities observed for HII and LINER but lower than typical Seyfert 2 luminosities. One possible explanation would be that the source is a highly absorbed AGN with an equivalent hydrogen column of $10^{24} \mathrm{~cm}^{-2}$ or more, which would explain the low value measured for the index of the power law. We studied the starforming activity of the source. Based on the hard luminosity, the SFR derived is $13 M_{\odot} / \mathrm{yr}$. The SFR of AM1211-465SW is higher than the values derived with the same method for the classified pure starburst galaxies, i.e. AM0707-273W and both members of AM2040-674, pointing to the possibly LLAGN nature of this source. In any case, there is no strong evidence of the presence of a luminous AGN in AM1211-465SW and the X-ray activity is very likely due to a low-luminosity AGN or a very intense starburst activity.

\subsection{Activation of quiescent black holes}

It is widely accepted that optical spectroscopy alone can provide a misleading classification of galactic nuclei. Indeed, an extensive study performed with Chandra (Maiolino et al. 2003) revealed that the density of AGN is twice the one estimated from optical spectroscopic surveys. This misclassification in the optical studies is associated with their high sensitivity to obscuration. Interestingly, in merging systems where the presence of obscuring gas is expected, the detection of elusive AGN is about $50 \%$ of the studied systems in the X-ray band. The most outstanding example of a binary AGN was discovered by Komossa et al. (2003) based on Chandra observation of the ultraluminous galaxy NGC 6240. Ballo et al. (2004) suggest that both galaxies of the merger Arp 299 host an AGN-type nucleus. Both galaxies were spectroscopically classified as starforming galaxies or LINER based on optical and mid-IR data. More recently, Guainazzi et al. (2005) observed ESO509-IG066 with XMM-Newton. The merger system hosts two AGN with $\mathrm{X}$-ray luminosities of $\sim 10^{43} \mathrm{erg} / \mathrm{s}$. One of the galaxies of the pair was misclassified as an HII galaxy based on optical and IR observations (Sekiguchi \& Wolstencroft 1992). In this work, the X-ray properties of the SW component of the AM1211-465 pair suggest an AGN nature for its nucleus. If this is the case, AM1211-465 would be the fourth example of an AGN pair in a merging system.

Exhaustive studies of known quasar pairs reveal that the galaxies, when merging, could become active (Mortlock et al. 1999). The model suggests that when the galaxies reach a certain distance, tidal interactions cause gas flowing into the cores of the galaxies switching on the black holes. Taking into account 16 good candidates to binary quasars, Mortlock et al. (1999) estimated this activation distance to be in the range between 50 to $100 \mathrm{kpc}$. When the merger becomes more stable, at typical distances of $10 \mathrm{kpc}$, the inflow of gas would cease and the back holes become quiescent again. The activation distance range estimated in Mortlock et al. (1999) is based on projected physical separation when considering different cosmological models. The authors therefore expect that the binary quasars exist in very distorted hosts and not in relaxed mergers. The assessment of the critical distance is important for understanding the processes that lead to nuclear activity in interacting galaxies. According to this model, the activation the the two nuclear black holes can only occur in encounters of galaxies with similar sizes, since the interaction between dwarf and large galaxies would result in a single AGN. However, only a small fraction of quasars have a second nearby quasar because in general the pairs are in reality gravitational lenses or chance alignments. Therefore, only a small number of true (or good) candidates of quasar pairs have been identified so far and most of them have high redshifts $(z=1-2.5)$.

As a matter of fact, X-ray studies are efficient at discovering hidden pairs of AGN. Due to a selection effect, all AGN pairs discovered in the X-ray band are nearby objects. In these cases, we are therefore able to determine the properties of the host galaxies, which in many of the distant pairs are not even detected. The three AGN pairs detected in the X-rays, NGC 6240, Arp299, and ESO509-IG066, have an apparent separation distance in the range of 1.4-13 kpc, lower or close to the minimum value of the activation distance, i.e. $10 \mathrm{kpc}$. Only the host galaxies of the two former mergers present clearly disrupted shapes.

In this work, we have detected a possibly double AGN system, AM1211-465. The apparent separation of the galaxies is $98 \mathrm{kpc}$, so in this case, close to the upper limit of the activation distance. Finally, contrary to the Mortlock hypothesis, no sign of disruption is observed in the X-ray images of any of the two host galaxies of AM1211-465.

\subsection{Comparison with other merging galaxies}

Excluding AM1211-465NE and AM1211-465SW, the derived properties of the rest of the objects are compatible with those found in other interacting systems where no evidence of AGN has been found (Jenkins et al. 2005, and references therein). In general, the X-ray spectrum of the interacting galaxies is characterized by a power law and a single or a double thermal component present in the soft energy band. The X-ray $(0.3-10 \mathrm{keV})$ luminosities are in the range $(0.2-5) \times 10^{41} \mathrm{erg} / \mathrm{s}$ and are explained by the intense starforming activity triggered by the merging process. High-resolution Chandra X-ray images show that many of the objects host luminous and ultraluminous X-ray sources (ULX). Brassington et al. (2005) find seven ULX out of 16 point-like sources spread in the merging system Arp270, and similar results are also observed in other mergers such as NGC 7714/NGC 7715 (Smith et al. 2005) and Arp299 (Ballo et al. 2004; Zezas et al. 2003). The limited spatial resolution of our XMM-Newton observation did not allow the detection of possibly off-nuclear point-like X-ray sources. Higher resolution images would help to unambiguously determine where the hard X-ray emission of AM1211-465SW and AM0707-273E originate. Jenkins et al. (2005) find that the XMM-Newton spectrum of NGC 7771 is compatible with being due to an AGN, although the evidence is not strong. Its companion galaxy, NGC 7770, 
is classified as a Starburst galaxy. In this sense, even if only the NE component of the AM1211-465 system is an AGN, the detection favors the role of fueling quiescent supermassive black holes in merging systems and the importance of systematic X-ray studies to unveil the AGN, obscured by the merging process.

\section{Conclusions}

In this paper we have presented the X-ray imaging and spectral analysis of three pairs of interacting galaxies, AM0707-273, AM1211-465, and AM2040-674, observed with XMM-Newton. Six galaxies were detected and the XMM-Newton spatial resolution allowed us to isolate each member of the pairs.

The images of the galaxies show nuclear and extended diffuse X-ray emission for all pairs. All the galaxies, except AM2040-675S, were detected in the hard band. No evidence of off-nuclear point-like sources were observed in any of the galaxy pairs. However, the coarse spatial resolution of the EPIC cameras on-board XMM-Newton prevents us from making strong statements on this point. Hints of merging processes were observed for AM0707-273 and AM1211-465 in the form of intergalactic soft X-ray emitting gas. The apparent separation of the two AM2040-674 galaxies does not allow us to disentangle the emission of the host galaxies from the emission due to possible disruption of the merging process.

For five (the exception being AM2140-674S) out of the six galaxies, enough counts were detected to allow spectrum extractions and analysis. All the spectra analyzed are complex, and two components are needed to explain the $0.3-10 \mathrm{keV}$ emission. The best-fit model for all the sources consists of a power law, accounting for the bulk of the hard emission and a thermal mekal or bremsstrahlung component that explains the ubiquitously observed excess in the soft band. The luminosities in the $0.3-10 \mathrm{keV}$ band range from $(0.4-40) \times 10^{41} \mathrm{erg} / \mathrm{s}$.

Optical and IR spectroscopic studies previously classified the nuclear activity of all galaxies as powered by intense starforming processes. The XMM-Newton data allowed us to unambiguously unveil the AGN nature of AM1211-465NE, misclassified in the optical due to its high obscuration. The spectrum shows the marginal presence (98.8\% according to the F-test) of the neutral $\mathrm{FeK} \alpha$ line, reinforcing the AGN nature of the source. The spectral properties of its companion galaxy, AM1211-465SW, are compatible with the presence of an AGN. If this is the case, AM1211-465 would be the fourth example of a binary AGN discovered in X-rays. AM0707-273E could also host a low-luminosity AGN, although the evidence is not strong. The X-ray classification of the three remaining sources, AM0707-273W and both members of AM2040-674, as HII galaxies agrees with the results of other studies in optical/IR.

Our results do not contradict the theory of the activation of quiescent black holes through the gas accretion triggered by encounters of galaxies (Mortlock et al. 1999). Although, we have only found signatures of double AGN in one galaxy pair (AM1211-465), its projected members' distance $(\simeq 100 \mathrm{kpc})$ is comparable to the AGN activation radius. Our results also point to the distance between galaxies in merging systems not being the only determining parameter in the black hole activation. However, this type of analysis shows the importance of X-ray studies of galaxy pairs for accurately determining the nature of their nuclei, in particular in those suffering high absorption. Systematic and high-resolution X-ray surveys of galaxy pairs are important for furthering our understanding of the mechanisms that originate the nuclear activity of AGN, as well as for a complete demography of them.

Acknowledgements. The authors would like to thank the anonymous referee for the very useful comments that significantly improved the work. The authors would also want to thank Miguel Mass-Hess and Héctor Otí-Floranes for helpful discussions. E.J.B. and M.S.L. acknowledge funding from the Spanish MEC grant AYA2004-08260-C03-03. DRG acknowledges support by the Mexican research council (CONACyT) under the grant 49942.

\section{References}

Arnaud, K. A. 1996, Astronomical Data Analyis and Systems, ASP Conf. Ser., 101,17

Arnett, D. 1995, ARA\&A, 33, 115

Avni, Y. 1976, ApJ, 210, 642

Ballo, L., Braito, V., Della Ceca, R., et al. 2004, ApJ, 600, 634

Bennett, C. L., Halpern, M., Hinshaw, G., et al. 2003, ApJS, 148, 1

Brassington, N. J., Read, A. M., \& Ponman, T. J. 2005, MNRAS, 360, 801

Cappi, M., Persic, M., Bassani, L., et al. 1999, A\&A, 350, 777

Corbett, E. A., Kewley, L., Appleton, P. N., et al. 2003, ApJ, 583, 670

Dickey, J. M., \& Lockman, F. J. 1990, ARA\&A, 28, 215

Fouque, P., Durand, N., Bottinelli, L., Gouguenheim, L., \& Paturel, G. 1992, Catalogue of Optical Radial Velocities, 1, 1

Gabriel, C., et al. 2004, Astronomical Data Analysis Software and Systems (ADASS) XIII, ASP Conf. Ser., 314, 759

Gonzalez-Martin, O., Masegosa, J., Marquez, I., Guerrero, M. A., \& Dutzin-Hacyan, D. 2006 [arXiv: astro-ph/0605629]

Grimm, H.-J., Gilfanov, M., \& Sunyaev, R. 2003, MNRAS, 339, 793

Guainazzi, M., Piconcelli, E., Jiménez-Bailón, E., \& Matt, G. 2005, A\&A, 429, L9

Hennawi, J. F., Strauss, M. A., Oguri, M., et al. 2006, AJ, 131, 1

Hewett, P. C., Foltz, C. B., Harding, M. E., \& Lewis, G. F. 1998, AJ, 115, 383

Ho, L. C., Feigelson, E. D., Townsley, L. K., et al. 2001, ApJ, 549, L51

Jansen, F., Lumb, D., Altieri, B., et al. 2001, A\&A, 365, L1

Jenkins, L. P., Roberts, T. P., Ward, M. J., \& Zezas, A. 2004, MNRAS, 352, 1335

Jenkins, L. P., Roberts, T. P., Ward, M. J., \& Zezas, A. 2005, MNRAS, 357, 109

Jiménez-Bailón, E., Piconcelli, E., Guainazzi, M., et al. 2005, A\&A, 435, 449

Jogee, S., Scoville, N., \& Kenney, J. D. P. 2005, ApJ, 630, 837

Kendall, M. G., \& Stuart, A. 1973, The advanced Theory of Statistics, Vol. 2 (New York: Hafner), Sect. 19.26, 97

Kennicutt, R. C., Jr. 1998, ApJ, 498, 541

Kochanek, C. S., Falco, E. E., \& Muñoz, J. A. 1999, ApJ, 510, 590

Komossa, S., Burwitz, V., Hasinger, G., et al. 2003, ApJ, 582, L15

Kormendy, J., Bender, R., Magorrian, J., et al. 1997, ApJ, 482, L139

Maiolino, R., Comastri, A., Gilli, R., et al. 2003, MNRAS, 344, L59

Morrison, R., \& McCammon, D. 1983, ApJ, 270, 119

Mortlock, D. J., Webster, R. L., \& Francis, P. J. 1999, MNRAS, 309, 836

Persic, M., \& Rephaeli, Y. 2002, A\&A, 382, 843

Piconcelli, E., Jimenez-Bailón, E., Guainazzi, M., et al. 2004, MNRAS, 351, 161

Sekiguchi, K., \& Wolstencroft, R. D. 1992, MNRAS, 255, 581

Shlosman, I. 2005, AIPC, 783, 223

Smith, B. J., Struck, C., \& Nowak, M. A. 2005, AJ, 129, 1350

Strauss, M. A., Huchra, J. P., Davis, M., et al. 1992, ApJS, 83, 29

Strickland, D. K., Heckman, T. M., Colbert, E. J. M., Hoopes, C. G., \& Weaver, K. A. 2004, ApJS, 151, 193

Theureau, G., Coudreau, N., Hallet, N., et al. 2005, A\&A, 430, 373

Wong, O. I., Ryan-Weber, E. V., Garcia-Appadoo, D. A., et al. 2006, MNRAS, 371,1855

Zezas, A., Ward, M. J., \& Murray, S. S. 2003, ApJ, 594, L31 